\title{
A simple method of placing a coronary sinus catheter through the femoral vein in miniature swine
}

\author{
YUPENG BAI, LIQUN HU, DELONG YU, SHENG PENG, MINGJING ZHANG, \\ XIAOGANG LIU and YE GU \\ Department of Cardiology, Puai Hospital, Huazhong University of Science and Technology, \\ Wuhan, Hubei 430033, P.R. China
}

Received December 8, 2015; Accepted December 9, 2016

DOI: $10.3892 /$ etm.2017.4158

\begin{abstract}
The aim of the present study was to evaluate the feasibility of placing a coronary sinus (CS) catheter through the femoral veins of miniature swine. A total of 16 male domestic pigs (3-4 months old, $25 \pm 2 \mathrm{~kg}$ ) were used. Firstly, the anatomic structure of the CS ostium of swine heart was observed at different angles under X-ray. The guide wire and Cobara catheter were subsequently advanced into the right atrium through the femoral vein. Subsequently, the guide wire was retracted behind the fix curve of the Cobara catheter and the catheter bent spontaneously in the absence of supporting guide wire following retraction. The catheter was then gently rotated clockwise to direct the catheter tip to the left allowing the catheter to easily be placed in the CS ostium. This method was associated with a short procedure time: The time on separation of the blood vessels was $15.5 \pm 5.8 \mathrm{~min}$ and the time of radiation exposure was $112 \pm 20 \mathrm{sec}$. The success rate of placing the catheter to CS ostium was $100 \%$. Only one pig experienced a hematoma after the sheath was pulled out. All swine recovered without serious complications, such as perforation of coronary vein and pericardial tamponade. Therefore, this method of placing CS catheter is simple, safe and reliable, which may offer help for related research.
\end{abstract}

\section{Introduction}

Pigs have similar coronary artery anatomical features to humans, thus pigs are often used in coronary intervention studies as an ischemia/reperfusion model through the use of balloon obstruction (1), coronary microemboli model (2) and coronary slow flow model (3) through microsphere injection. Pigs are also used in electrophysiological and pacemaker studies such as in radiofrequency ablation (4) and cardiac

Correspondence to: Professor Ye Gu, Department of Cardiology, Puai Hospital, Huazhong University of Science and Technology, 473 Hanzheng Street, Wuhan, Hubei 430033, P.R. China

E-mail: yegu2003cn@163.com

Key words: coronary sinus, swine, femoral vein resynchronization therapy (CRT) research (5). Placing a coronary sinus (CS) catheter or electrode in the coronary sinus ostium is an essential technique in these studies. However, placing CS catheters in swine remains a challenging technique for researchers. Previous studies have collected metabolic and hemodynamic measurements data through CS catheters with the open-chest technique in pigs (6-11), and have demonstrated that the aforementioned method islinked with complicated operation procedures and heavy traumatic injury in swine. A number of novel techniques have been developed to place the CS catheter in swine. Hilbert et al (12) introduced a method utilizing fluoroscopy and a high-density electroanatomical mapping catheter to verify the CS anatomy to facilitate CS catheter placement. Neizel et al (13) reconstructed the cardiac venous system in pigs using magnetic resonance imaging and performed magnetic resonance-guided placements of CS catheters in swine. Nazarian et al (14) used a flexible steerable fiber optic infrared endoscope to visualize the CS ostium and branches in closed-chest dogs. However, such methods require high-end equipment, which is not available in all laboratories. Therefore, in the present study, the efficacy of a simple CS catheter placement method via the femoral vein in a closed-chest pig model was assessed.

\section{Materials and methods}

Animals. A total of 16 male domestic pigs (3-4 months; $25 \pm 2 \mathrm{~kg}$ ) were used in this study, which were purchased from the Experimental Animal Center of Tongji Medical College, Huazhong University of Science and Technology (Wuhan, China). Aspirin (2-3 mg/kg/day; Bayer China Co., Ltd., Shanghai, China) was mixed in the food three days prior to experimental studies. Animals were maintained in a conventional animal environment $\left(21 \pm 2^{\circ} \mathrm{C} ; 55 \pm 10 \%\right.$ humidity), with a 12-h light/dark cycle (lights off at 19:30 h and no twilight period). Animals had free access to water and were fed three times per day in accordance with the Guide for the Care and Use of Laboratory Animals published by the US National Institute of Health (Bethesda, MD, USA) (15). Four animals were used in the pilot study for initial evaluation of the surgical technique and 12 animals were used in the main study. The study protocol was approved by the Tongji Medical 
College Council of the Animal Care Committee of Huazhong University of Science and Technology (Wuhan, China).

Preoperative preparation and anesthesia. The pigs fasted for $12 \mathrm{~h}$ prior to operation but had ad libitum access to drinking water until $4 \mathrm{~h}$ prior to operation. Pigs were anesthetized by an intramuscular injection of ketamine $(15 \mathrm{mg} / \mathrm{kg}$; Jiangsu Hengrui Medicine Co., Ltd., Lianyungang, China) combined with atropine (1 mg; Guangdong South China Pharmaceutical Co., Ltd., Guangzhou, China), then fixed in a supine position on the workstation. During the operation, 3-5 $\mathrm{ml} 3 \%$ pentobarbital sodium solution (Sigma-Aldrich; Merck Millipore, Darmstadt, Germany) was injected via ear marginal vein on demand to maintain the anesthesia state. Electrocardiogram and vital signs were continuously monitored. Oxygen saturation $\left(\mathrm{SO}_{2}\right)$ was measured with a pulse oximeter attached to the ear of pigs.

Evaluation of the surgical technique. In the pilot study, four pigs were used to evaluate the feasibility of the surgical technique applied. A catheter was inserted into the CS through the jugular vein of the pigs; however, it took a long time to separate the neck vasculature due to the complexity of the anatomical structures. Therefore, the method of catheter insertion though the femoral vein was assessed during the pilot study and main study. Following routine disinfection, the muscle layer and the femoral sheath, femoral vein, arteria cruralis and nerve were carefully separated. A 5F vascular sheath (Cordis Corporation, Milpitas, CA, USA) was placed for arterial and venous access. Anticoagulation was induced with $200 \mathrm{IU} / \mathrm{kg}$ heparin sodium (Jiangsu Wanbang Biopharmaceuticals Co., Ltd., Xuzhou, China). The arterial sheath can be used for pressure monitoring, coronary angiography (16). The 5F pigtail catheter (Cordis Corporation, Milpitas, CA, USA) was advanced into the right atrium from the right femoral vein. Right atrium imaging was performed [right anterior oblique (RAO) $30^{\circ}$, anterior-posterior (AP) angle)] to observe the CS opening and record right atrium pressure.

Imaging analysis of pig heart. Under the X-ray, the transparent triangle area in the pig hearts could not be clearly visualized at RAO $30^{\circ}$, left anterior oblique (LAO) $45^{\circ}$, AP angle, as it is in humans. In order to observe the structure of the right atrium and the CS ostium, the 5F pigtail catheter was advanced to the lower part of right atrium and radiography was performed (AP, RAO $30^{\circ}$ angle) with a high pressure injector $(20 \mathrm{ml} / \mathrm{sec}$; $300 \mathrm{kPa}$; Auto injector 120S; Nemoto Co., Ltd., Tokyo, Japan). The CS ostium could clearly be detected at AP or RAO $30^{\circ}$, and the contorts of CS could be clearly presented at the AP position (Fig. 1A and B).

Radiation dose assessment. All operations were performed by two experienced researchers. Radiation exposure time was recorded and the radiation dose received by the operator was detected using a Wearing X-ray detector provided by the Wuhan Center for Disease Prevention and Control.

\section{Results}

CS catheter placement method. In the main experiment, a 5F guide wire (RF*GA3513M; Terumo Corporation, Tokyo,
Japan) and Cobara catheter (RF*DB55008M; Terumo Corporation, Tip curve L: Middle) were advanced into the right atrium through the femoral vein and the guide wire was retracted out of the catheter, which then spontaneously bent. CS ostium was generally located one width of a rib under the middle of long axis of the heart. Following gentle clockwise rotation of the catheter to the left, the catheter could be easily and smoothly placed into the CS ostium. Following successful placement, the characteristic swing sign could be visualized. Contrast medium was injected to confirm the successful placement of catheter inside the CS (Fig. 1C and D). Afterwards, CS blood sampling was performed (Fig. 1E and F).

Reliability and safety evaluation. This method was associated with a short procedure time (the time used on separation of the blood vessels was $15.5 \pm 5.8 \mathrm{~min}$ and the time of radiation exposure was $112 \pm 20 \mathrm{sec}$ ) and a success rate of $100 \%$. Only one pig experienced a hematoma after the arterial sheath was pulled out. All swine recovered without serious complications, including perforation of coronary vein and pericardial tamponade. Additionally, this method had a low per capita irradiation dose $(1.56 \pm 0.32 \mu \mathrm{Gy})$ at each operation.

\section{Discussion}

Cardiologists usually pay more attention to the arterial system of coronary circulation, and less to the coronary vein system. With the rapid development of interventional techniques in recent years, there is an increased requirement of CS placement of CS catheters, for example to determine the myocardial metabolic product in CS. Moreover, placing electrodes to the CS during electrophysiological examinations and left ventricular electrode placement in CRT also requires the placement of a CS catheter in selected patients. This technique is also necessary during retrograde stem cell transplantation through the CS route (17). It has been demonstrated that simultaneous coronary artery and CS perfusion facilitate myocardial preservation (18). As pigs are the ideal experimental animal model when investigating the heart, particularly in coronary intervention and electrophysiology pacemaker research field, an effective method for placing a CS catheter in swine is particularly important. The primary difficulty is that the catheter may pop out and injure the CS if the catheter is pushed directly without adjusting the direction. In some pigs, pushing catheters to distal CS is difficult. The current study demonstrated that if the guide wire can be fixed, and the direction of catheter gently adjusted, the majority of catheters can be advanced to the distal CS without injuring the CS. In addition, the COBARA catheter used in the present study is a $5 \mathrm{~F}$ peripheral vessel operated catheter with soft tip; the probability of perforation is thus low. Moreover, placing the CS catheter through the femoral vein may reduce the operation time and avoid pneumothorax and hemothorax complications. This method may also significantly reduce the radiation dose the operator is exposed to.

Placing a catheter to the CS through the femoral vein in miniature pigs may also be useful in electrophysiology research in swine animal models. If an adjustable curved electrode is used and the direction of the catheter is gently 
A

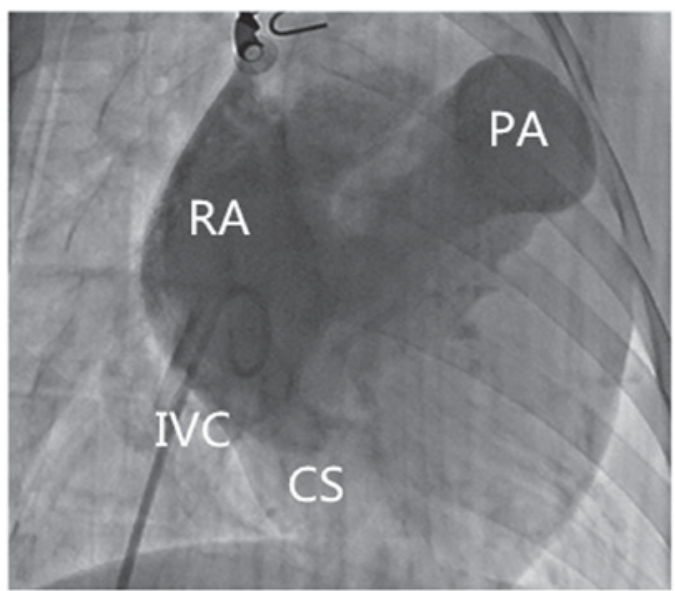

C

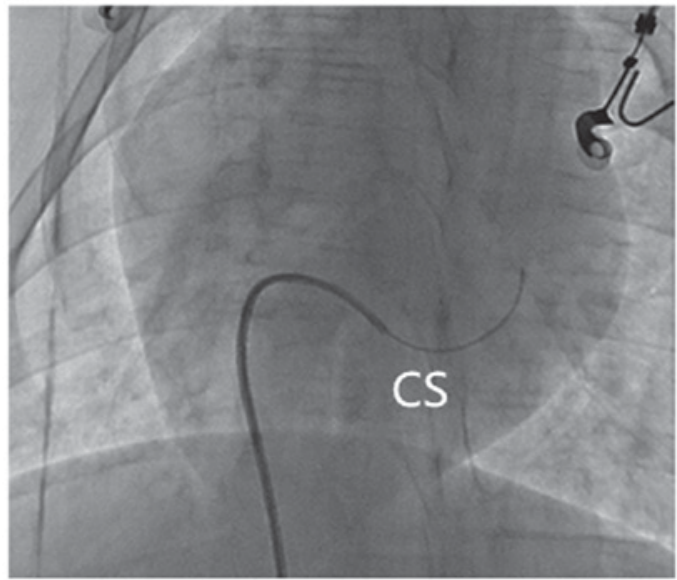

$\mathbf{E}$

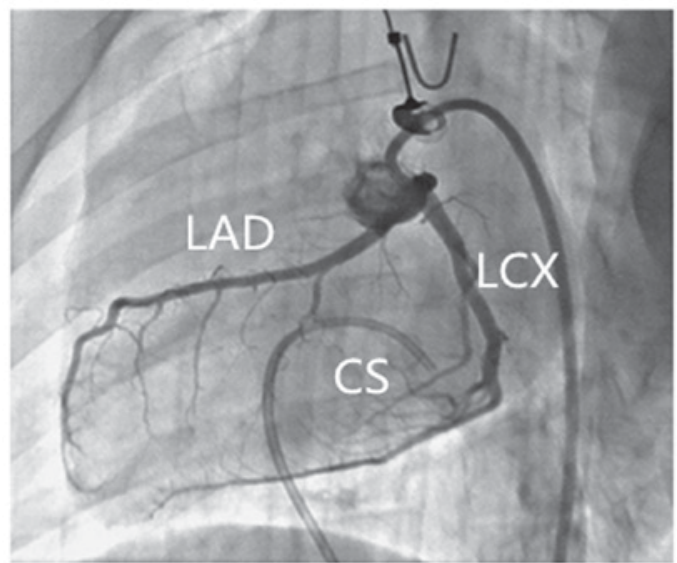

B

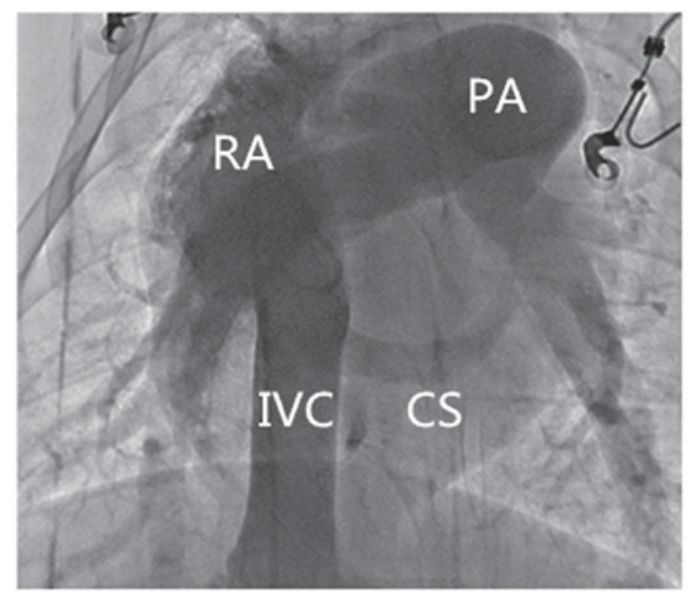

D

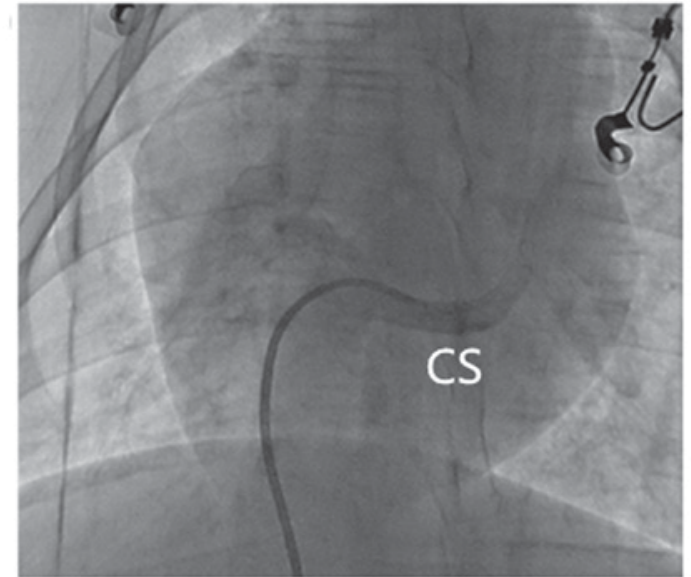

F

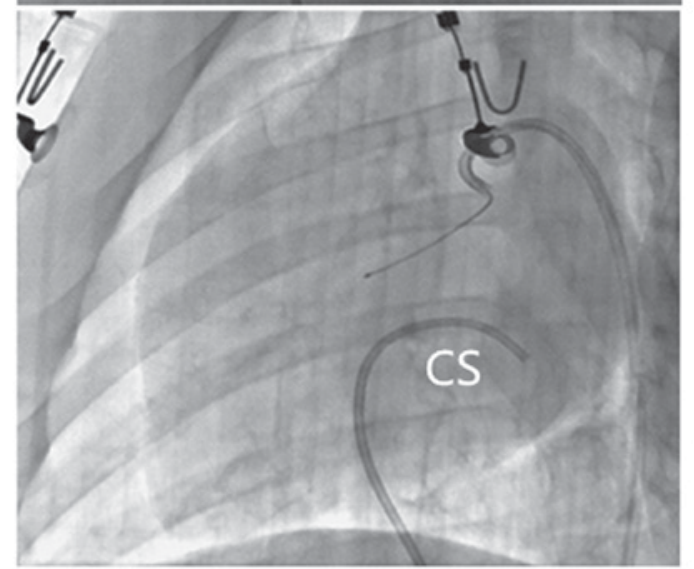

Figure 1. CS contorts and catheterplacementprocedures. (A) Right atrium imaging (RAO 30 ${ }^{\circ}$. (B) Right atrium imaging (AP). (C) Thread entry into the CS (AP). (D) CS imaging (AP). (E) Simultaneous placement of CS catheter and coronary arteriography (LAO 30 $0^{\circ}$. (F) Simultaneous placement ofCS catheter and guide wire in coronary $\left(\mathrm{LAO} 30^{\circ}\right.$ ). RA, right atrium; PA, pulmonary artery; IVC, inferior vena cava; LAD, anterior descending branch; LCX, left circumflex; $\mathrm{CS}$, coronary sinus; AP, anterior-posterior; LAO, left anterior oblique; RAO, right anterior oblique.

adjusted, the CS electrode could successfully be advanced to the distal CS.

Placing the CS catheter through the femoral vein in miniature pigs has the following advantages: i) Avoiding open chest and other complex surgical procedures, thus reducing trauma and the operating time; ii) does not require the support of endotracheal intubation and breathing machine ventilation when animals are under anesthetic; and iii) avoiding the jugular vein puncture-related pneumothorax and hemothorax complications. The groin hematoma complication observed in one of the pigs in the current study may be avoided by prolonged local oppression.
In conclusion, the method of lacing a catheter into the CS of swine assessed in the current study is simple, safe and reliable; it may be beneficial for scientific researchers using swine as animal models in related coronary studies.

\section{Acknowledgements}

The present study was supported by the National Natural Science Foundation of China (grant no. 81270266) and the Nature Science Foundation of Hubei Province (grant nos. 2014CFB365 and 2015CFA081). 


\section{References}

1. Kondo K, Shibata R, Unno K, Shimano M, Ishii M, Kito T, Shintani S, Walsh K, Ouchi N and Murohara T: Impact of a single intracoronary administration of adiponectin on myocardial ischemia/reperfusion injury in a pig model. Circ Cardiovasc Interv 3: 166-173, 2010.

2. Ma J, Qian J, Ge J, Zeng X, Sun A, Chang S, Chen Z and Zou Y: Changes in left ventricular ejection fraction and coronary flow reserve after coronary microembolization. Arch Med Sci 8: 63-69, 2012.

3. Hu L, Bai Y, Gu Y, Yu D, Peng S, Liu X, Zhang M, Liu T and $\mathrm{Hu}$ S: Establishment of an experimental angiographic slow coronary flow model by microsphere embolism in swines. Int $\mathbf{J}$ Cardiol 176: 1123-1125, 2014.

4. Bessiere F, N'djin WA, Colas EC, Chavrier F, Greillier P, Chapelon JY, Chevalier P and Lafon C: Ultrasound-guided transesophageal high-intensity focused ultrasound cardiac ablation in a beating heart: A pilot feasibility study in pigs. Ultrasound Med Biol 42: 1848-1861, 2016.

5. Rigol M, Solanes N, Fernandez-Armenta J, Silva E, Doltra A, Duchateau N, Barcelo A, Gabrielli L, Bijnens B, Berruezo A, et al: Development of a swine model of left bundle branch block for experimental studies of cardiac resynchronization therapy. J CardiovascTransl Res 6: 616-622, 2013.

6. Safranow K, Rzeuski R, Listewnik MJ, Jakubowska K, Rać ME, Olszewska M and Chlubek D: Myocardial and coronary sinus purines as indicators of pig heart energy metabolism during reperfusion after extracorporeal circulation. Acta Physiol Scand 185: 13-23, 2005.

7. Karapanos NT, Wettstein PJ, Li Z, Huebner M, Park SJ, Deschamps $\mathrm{C}$ and Cassivi SD: Does lung ischemia and reperfusion have an impact on coronary flow? A quantitative coronary blood-flow analysis with inflammatory cytokine profile. Eur J Cardiothorac Surg 41: 154-161, 2012.

8. Pantely GA, Bristow JD, Ladley HD and Anselone CG: Effect of coronary sinus occlusion on coronary flow, resistance, and zero flow pressure during maximum vasodilatation in swine. Cardiovasc Res 22: 79-86, 1988.
9. Andersen FR, Sejersted OM and Ilebekk A: A model for quantitative sampling of myocardial venous blood in the pig. Acta Physiol Scand 119: 187-195, 1983.

10. Harvey RC and Jones EF: A technique for bioinstrumentation of the thorax of miniature swine. Lab Anim Sci 32: 94-96, 1982.

11. McKenzie JE, Scandling DM, Ahle NW, Bryant HJ, Kyle RR and Abbrecht PH: Effects of soman (pinacolyl methylphosphonofluoridate) on coronary blood flow and cardiac function in swine. Fundam Appl Toxicol 29: 140-146, 1996.

12. Hilbert S, Kosiuk J, John S, Hindricks G and Bollmann A: A guide to the porcine anatomy for the interventional electrophysiologist. Fluoroscopy and high density electroanatomical mapping. J Cardiovasc Transl Res 8: 67-75, 2015.

13. Neizel M, Krämer N, Schütte A, Schnackenburg B, Krüger S, Kelm M, Günther RW, Kühl HP and Krombach GA: Magnetic resonance imaging of the cardiac venous system and magnetic resonance-guided intubation of thecoronary sinus in swine: A feasibility study. Invest Radiol 45: 502-506, 2010.

14. Nazarian S, Knight BP, Dickfeld TL, Zviman MM, Jayanti VB, Amundson D, Hanlin J, Castleberry J, Smith MF, Blankenship L, et al: Direct visualization of coronary sinus ostium and branches with a flexible steerable fiberoptic infrared endoscope. Heart Rhythm 2: 844-848, 2005.

15. Guide for the Care and Use of Laboratory Animals, NIH Publication No. 85-23, revised 1996.

16. Ma J, Qian J, Chang S, Chen Z, Jin H, Zeng M, Zou Y and Ge J: Left ventricular remodeling with preservedfunction after coronary microembolization: The effect of methylprednisolone. Eur J Med Res 19: 7, 2014

17. Berry MF, Engler AJ, Woo YJ, Pirolli TJ, Bish LT, Jayasankar V, Morine KJ, Gardner TJ, Discher DE and Sweeney HL: Mesenchymal stem cell injection after myocardial infarction improves myocardial compliance. Am J Physiol Heart Circ Physiol 290: H2196-H2203, 2006.

18. Tian G, Xiang B, Dai G, Sun J, Lindsay WG and Deslauriers R: Simultaneous antegrade/retrograde cardioplegia protects myocardium distal to a coronary occlusion: A study in isolated pig hearts. Magn Reson Med 46: 773-780, 2001. 